Recepción: 20 / 04 / 2017

Aceptación: 20 / 05 / 2017

Publicación: 15 / 07 / 2017

Ciencias de la Educación

Artículo Científico

\title{
Detección temprana del retraso del lenguaje en niños menores de 6 años
}

\author{
Early detection of language delay in children under 6 years of age
}

\section{A detecção precoce de atrasos de linguagem em crianças com menos de 6 anos}

Viviana P. Patiño-Zambrano ${ }^{\mathrm{I}}$ viviana.patinoz@ug.edu.ec

Marìa D. Enireb-García II maria.enirebg@ug.edu.ec

Alexandra M. Alvarado-Alvarez III alexandra.alvaradoa@ug.edu.ec

Walter A. Patiño-Zambrano IV alejo8585@hotmail.com

Correspondencia: viviana.patinoz@ug.edu.ec

Magister en Gerencia de Salud para el desarrollo local; Universidad de Guayaquil, Guayaquil, Ecuador. Magister en Diseño Curricular; Universidad de Guayaquil, Guayaquil, Ecuador.

Magister en Gerencia de Servicios de Salud; Universidad de Guayaquil, Guayaquil, Ecuador.

Docente, Universidad de Guayaquil, Guayaquil, Ecuador. 


\section{Resumen}

En la ciudad de Chone, Manabí-Ecuador se realizó un proyecto de acción titulado "Detección temprana del retraso del lenguaje en niños menores de 6 años en el Hospital Napoleón Dávila Córdova de la ciudad de Chone provincia de Manabí año 2013.” Por medio de un diagnóstico situacional realizado con la técnica de grupo focal, con el personal de salud se identificó como problema principal que existe una escasa detección temprana de retraso en el lenguaje en los niños/as menores de 6 años. Como factores causales se identificó que el equipo de salud está desactualizado sobre la detección temprana del retraso en el lenguaje, que existe un área desorganizada, e inexistencia de coordinación institucional. Como respuesta a esta problemática se ejecutó un proyecto de acción cuya finalidad fue disminuir los trastornos del lenguaje mediante la detección temprana de retraso en lenguaje en niños/as menores de 6 años, la capacitación al equipo de salud, organización del área y coordinación institucional.

Palabras Clave: Trastornos en el lenguaje; retraso del lenguaje; detección; programa. 


\begin{abstract}
In the town of Chone, Manabí - Ecuador project entitled action was performed "Early detection of language delay in children under 6 years in the Hospital Napoleon Davila Cordova City Chone Manabí province in 2013. "Through a situational analysis conducted with the focus group technique, with health personnel was identified as the main problem that exists early detection of language delay in children / as children under 6 years. As causal factors are identified that the health team outdated about early detection of language delay, there is a disorganized area, and lack of institutional coordination. In response to this problem, an action project whose purpose was to reduce language disorders through early detection of language delay in children / as children under 6 years, training the health team, organization and institutional coordination area was run.
\end{abstract}

Keywords: Language disorders; language delay; detection; program. 


\section{Resumo}

Na cidade de Chone, Manabi-Equador um projeto de acção intitulado "atraso de linguagem de detecção precoce em crianças com menos de 6 anos em Cordova Davila Napoleão Hospital na cidade da província de Chone de Manabi 2013" foi realizada através de uma análise situacional realizado com a técnica de grupo focal, com o pessoal de saúde foi identificado como o principal problema que há pouco a detecção precoce de atraso de linguagem em crianças / as crianças menores de 6 anos. Identificados como fatores causais que a equipe de saúde está desatualizado sobre a detecção precoce de atraso de linguagem, há uma área desorganizada, e falta de coordenação institucional. Em resposta a este problema um projeto de ação cujo objetivo era reduzir distúrbios de linguagem através da detecção precoce de atraso de linguagem em crianças / as crianças menores de 6 anos, a formação da equipe de saúde, a organização área e coordenação institucional foi executado.

Palavras-chave: Distúrbios da linguagem; atraso de linguagem; a detecção; o programa. 


\section{Introducción.}

El trastorno del lenguaje es un problema que está presente en el Cantón Chone, Parroquia Chone, Provincia de Manabí, el más común en los niños, y el más conocido y fácil de identificar. Suele presentarse entre los tres y los cinco años de edad, con alteraciones en la articulación de los fonemas.

En Latinoamérica," La prevalencia de problemas auditivos entre los niños menores de 14 años es del 1,6\%; en los adultos de 15 años es del 8\%; y entre los mayores de 65 años del 38,62\%. Las infecciones del oído son las principales causas de las deficiencias auditivas y lo que da como consecuencia retrasos en el lenguaje y otras alteraciones cognitivas".(OMS, 2010)

La mayoría de las causas de pérdida de audición son fácilmente evitables porque muchas pueden ser tratadas gracias a la diagnosis temprana e intervenciones rápidas; la OMS menciona: "Falta conciencia sobre las causas, conocimiento para detectar las deficiencias y asumir que es necesario pedir ayuda para un problema que puede solucionarse. Cuando más demanda de servicios de asistencia haya, más se desarrollarán estos servicios". (OMS, 2010)

Dentro de las afecciones de la comunicación oral que precisan de un diagnóstico precoz y una intervención temprana para obtener resultados adecuados y definitivamente un buen pronóstico, se encuentran principalmente el retardo del desarrollo del lenguaje, de etiología secundaria a otras afecciones que en muchos casos constituyen el motivo de la consulta médica, por ser el lenguaje una de las áreas más afectadas, cuyas causas pueden ser el retraso mental, la hipoacusia, el autismo infantil etc. También existen otras causas que provocan el retardo del desarrollo del lenguaje como el bilinguísmo, la pobre estimulación verbal o la sobreprotección, pero que son de mejor pronóstico que las primeras mencionadas. 
Si el lenguaje no se desarrolla de forma normal pueden existir graves afectaciones para el desarrollo del niño no solo en la comunicación sino en todos los niveles: biológico, psicológico y socio familiar por eso la importancia de tener un diagnóstico a tiempo del retraso del lenguaje, para brindar el tratamiento adecuado. Siendo Manabí una de las provincias de mayor importancia en cuanto al número de habitantes y en relación a la estadísticas de discapacidad realizadas en los últimos años donde prevalecen la discapacidad auditiva que después de la discapacidad física se encuentran en segundo lugar afectando un gran porcentaje de la población y aumentando las alteraciones de lenguaje que deberían tener un tratamiento precoz.

En los hospitales del Ministerio de Salud en la Provincia de Manabí atienden a un gran número de niños/as con problemas auditivos en el año 2012 representaron el 5\% del total de las atenciones, en el Cantón Chone existen 8640 personas con discapacidades de las cuales 5175 son de la ciudad de Chone y 591 tienen discapacidades auditivas1 (INEC - Censo, 2010).

El proyecto constó de los siguientes objetivos: aplicar un programa de detección temprana de retraso en lenguaje en niños/as menores de 6 años, capacitar al equipo de salud sobre detección temprana del retraso del lenguaje en niños/ menores de 6 años para contribuir a la disminución de complicaciones; organizar un área para la detección temprana de problemas del lenguaje para un tratamiento deficiente e integral y coordinar con instituciones involucradas en la detección temprana del retraso en el lenguaje, para brindar a los padres de familia una orientación adecuada.

\section{Materiales y métodos.}

El trabajo fue realizado basados en la metodología heurística, con un diseño de campo, en el Hospital Napoleón Dávila Córdova de la ciudad de Chone provincia de Manabí, se usaron diversas herramientas como el marco lógico, el árbol de problemas y de objetivos, la observación 
participante con cuaderno de registro y la acción participativa dentro de la gestión para la atención de los niños menores de 6 años en el mencionado centro de salud.

A partir del árbol de problemas y objetivos se diseñó cada resultado esperado y se organizaron las actividades de observación y participación del proyecto.

\section{Resultados}

Resultado 1: Actualizar al equipo de salud sobre la detección temprana del retraso en el lenguaje de niños/as menores de 6 años

El objetivo primordial de la capacitación es la identificación temprana de niños/as con retraso en el lenguaje, y con riesgo de padecer alteraciones o trastornos de mayor riesgo, para que puedan ser remitidos a una evaluación diagnóstica completa que permita iniciar prontamente la intervención.

El interés por la identificación temprana conduce a una mejoría en el pronóstico de la mayoría de los niños y niñas con retraso en el lenguaje.

Los problemas de la detección temprana del retraso en el lenguaje, ocurre en diferentes ámbitos:

- A nivel familiar, los padres tienen dificultades para detectar los síntomas de retraso en el lenguaje, por la poca información recibida.

- En la salud, los profesionales de la atención primaria carecen de información y formación, tienden a pensar que son problemas leves o transitorios.

- En el ámbito educativo se da una situación desigual en la etapa infantil y principalmente del ciclo 0-6 años; de forma que donde se han desarrollado las escuelas infantiles y los equipos 
de atención Temprana, la sensibilidad y la experiencia en desarrollo evolutivo de los educadores asegura una detección mayor y más temprana.

- En el ámbito de los servicios sociales, porque los centros de desarrollo infantil (MIES), tiene limitada formación en trastornos específicos del desarrollo infantil en general.

- Los retrasos y trastornos del lenguaje son materias de gran importancia en el desarrollo del niño, el impacto a largo plazo de tales trastornos aumenta la importancia de las acciones para ayudar a los niños/as a adquirir el lenguaje. Los niños con trastornos tempranos del lenguaje están en riesgo de tener problemas sociales y conductuales, así como fracaso escolar.

Hasta el 3 de abril del 2013 el 100\% del Equipo de salud fue actualizado sobre el programa de detección temprana del retraso en el lenguaje de niños/as menores de 6 años.

\section{Grafico No. 1. Distribución porcentual de la encuesta de satisfacción a 21 participantes, sobre la capacitación de detección temprana del retraso en el lenguaje}

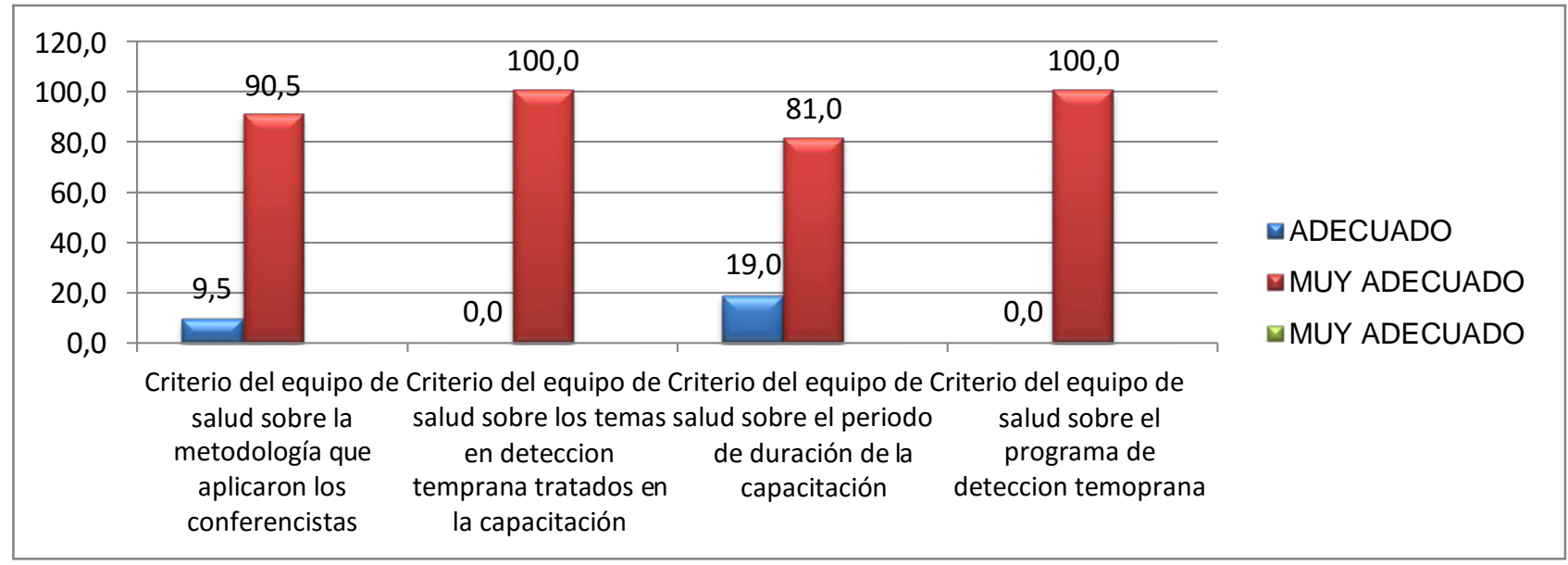

Elaborado por: Lcda. Viviana Patiño Zambrano

Fuente: encuesta de satisfacción 
Análisis e interpretación: En el gráfico $\mathrm{N}^{\mathrm{o}} 1$ podemos observar la valoración que le da 21 personas del equipo de salud a la capacitación, calificando como muy adecuado los temas en detección temprana, y el programa de detección temprana en un 100\%, el 90,5\% a metodología y el 81,0\% el periodo de duración de la capacitación.

Resultado 2: Organizar un área para la detección temprana de retraso en el lenguaje

\section{Socialización con autoridades}

El 2 de abril del 2013 realizó una reunión con los directivos y personal de salud al personal de salud para que expongan verbalmente los inconvenientes que se están dando para ejecutar eficazmente el programa, como resultado se detectó que no existe un área adecuada para posterior a la detección aplicar el debido tratamiento.

Los asistentes quedaron comprometidos en asignar y adecuar el área según los requerimientos

Planificación de las adecuaciones del área

Del 5 al 9 de mayo del 2013 por medio de la gestión realizada por el maestrante y equipo de salud en la adecuación del área se procedió a organizar el área a utilizar, se adquirieron los insumos, pinturas, sillas, escritorio, mesas, etc.

Gestión de insumos y materiales

El 21 de mayo del 2013 después de haber realizado la programación de insumos y materiales, fue entregada al jefe administrativo para la posteríos aprobación.

Adecuación del área para el tratamiento temprano el retraso en el lenguaje 
Se coordinó con el equipo de salud la organización y adecuación del área, mejorando sustancialmente las condiciones de trabajo, ya que un espacio adecuado para la atención infantil mejora las condiciones de progreso de los niños, por medio de la utilización de diversos materiales y herramientas, el acompañamiento del terapista de lenguaje y las familias. Estos elementos les proporcionan a los niños con algún retraso en el lenguaje lograr un avance en el sistema propioceptivo, Específicamente, cuando el espacio esta adecuado para estimular varios sentidos. De esta forma, se adquiere un conocimiento a través de un aprendizaje normal, sencillo y seguro para afianzar las habilidades de lenguaje y comunicación.

Para el cumplimiento del resultado se llevaron, se llevaron a cabo cuatro actividades que se cumplieron en un $100 \%$, como lo observamos en el siguiente cuadro y gráfico.

\section{Grafico No. 2 Distribución porcentual del cumplimiento de las actividades}

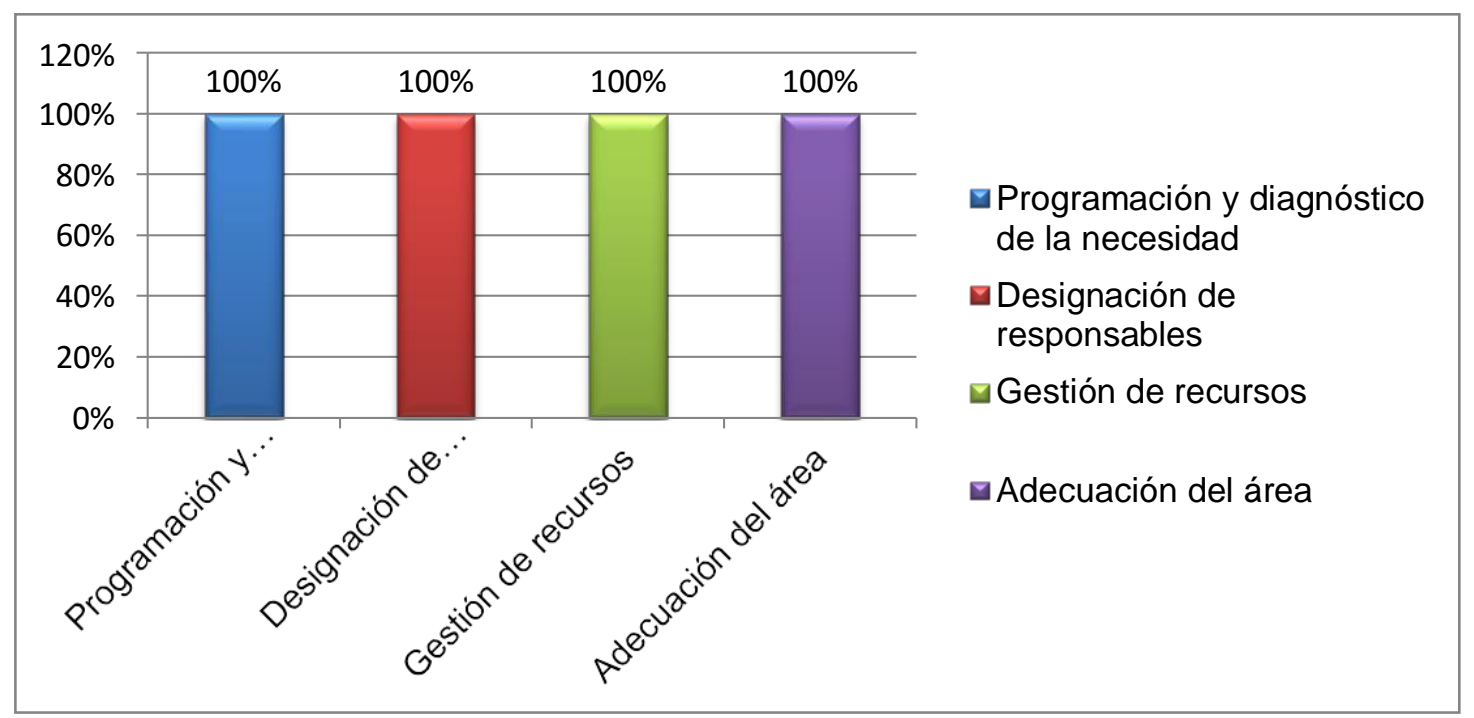

Fuente: Planificación de actividades

Elaborado por: Lcda. Viviana Patiño Zambrano 


\section{Gráfico No 3. Encuesta de satisfacción del usuario del área}

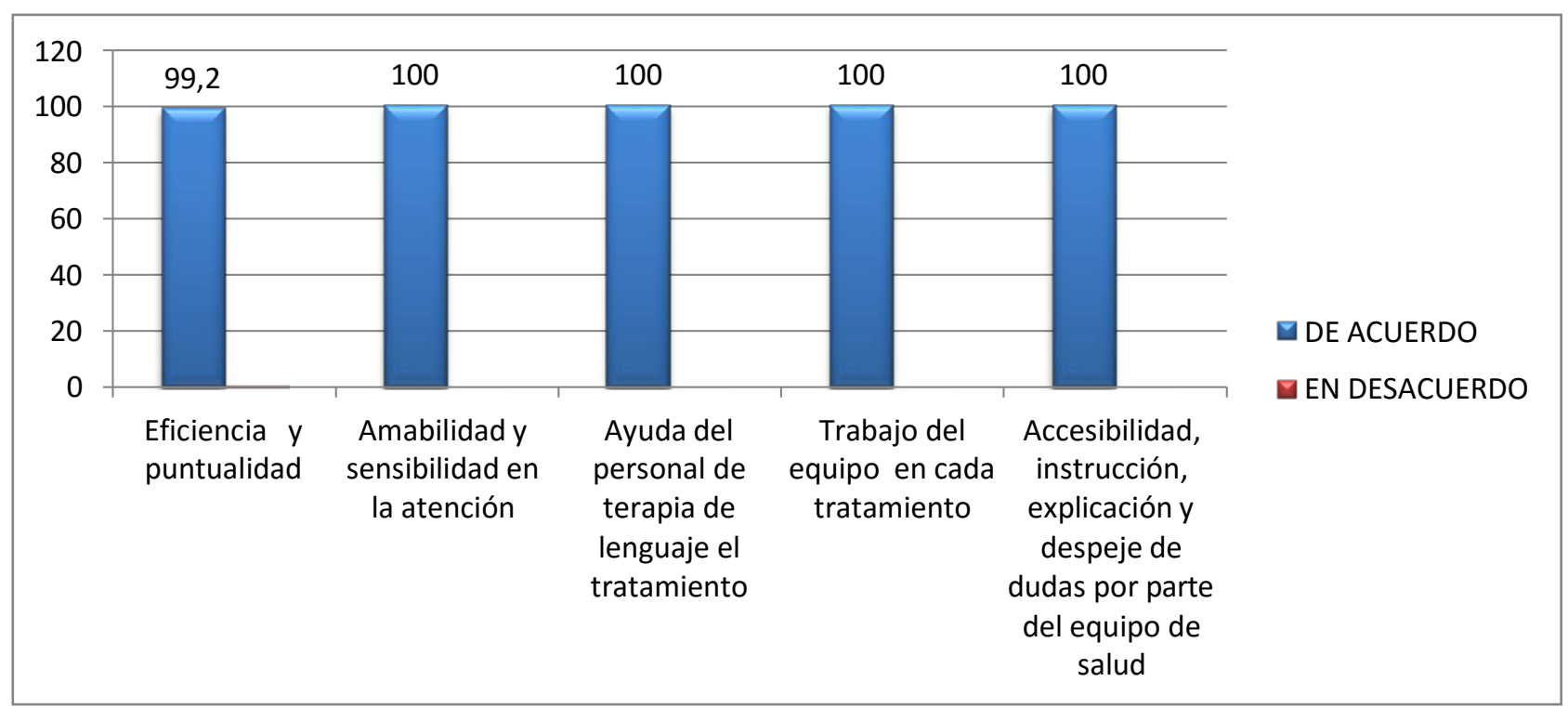

Fuente: Planificación de actividades

Elaborado por: Lcda. Viviana Patiño Zambrano

El gráfico No.3 nos indica que los usuarios están de acuerdo con la atención del área en cuanto a la amabilidad y sensibilidad en la atención ayuda del personal de terapia de lenguaje el tratamiento, trabajo del equipo en cada tratamiento, accesibilidad, instrucción, explicación y despeje de dudas por parte del equipo de salud en un $100 \%$, y un $99,2 \%$ en cuanto a eficiencia y puntualidad

Resultado 3: Existencia de coordinación interinstitucional en la detección temprana del retraso en el lenguaje

Diagnóstico de las instituciones involucradas

Para esta actividad se procedió a realizar una reunión con el equipo de salud en la que se evaluó la necesidad de incorporar a algunas instituciones de desarrollo infantil, se tomaron en cuenta 
10 instituciones que forman parte del MIES. Una vez seleccionadas se les convoco a las charlas para que sean partícipes del programa de detección temprana del retraso en el lenguaje, de esta manera ellos repliquen a los padres de familia sobre los signos de alarma,

\section{Estrategias de coordinación}

Son suficientemente conocidos los sucesivos estadios por los que, en general, transcurre el lenguaje infantil, pero estas adquisiciones en la infancia no se producen de manera lineal y uniforme, sino que pueden aparecer múltiples variables que dificulten dichos procesos $\mathrm{y}$, en consecuencia, perjudiquen el desarrollo global de las capacidades del sujeto. Es entonces cuando la atención temprana adquiere su máximo interés y se convierte en una técnica no ya necesaria, sino absolutamente imprescindible. La intervención temprana es un proceder terapéutico creado para ayudar a los niños con alteraciones del desarrollo, a mejorar estos trastornos o a moderar sus efectos. Se entiendo como intervención temprana en el lenguaje el conjunto de acciones encaminadas a desarrollar en los niños ( morfosintácticas, semánticas y pragmáticas necesarias a fin de facilitar los intercambios comunicativos de los más pequeños con su entorno.

Los niños susceptibles de este tipo de intervención proceden de 3 grupos específicos:

1) Niños en situación de riesgo biológico (prematuridad, bajo peso al nacer, asfixia, etc.)

2) Niños en situación de riesgo socioambiental (hijos de madres adolescentes, madres solteras, etc.)

3) Niños con retrasos, desviaciones o discapacidades del desarrollo ya establecidas (Análisis e interpretación: En la tabla y gráfico N$^{\circ} 6$ referente a la distribución porcentual del número de atenciones, en un grupo de 320 niños se logró establecer que al 100\% se le realizó la 
valoración médica y los factores de riesgos, solo al 105 se les realizó las audiometrías, el 47, $2 \%$ lo valoro el médico especialista, el $41,6 \%$ a terapias psicológicas y el 47,2\% se les aplico un esquema de tratamiento en terapia del lenguaje.

\section{Conclusiones.}

Que la implementación el programa de detección temprana de niños/as menores de 6 años por parte del equipo de salud, ha permitido a los niños/as con el apoyo de sus familiares, reduciendo los trastornos del lenguaje.

Se capacitó al 100\% del equipo de salud sobre detección temprana del retraso del lenguaje en niños/ menores de 6 años, contribuyendo a la disminución de complicaciones.

Se organizó un área para la detección temprana de problemas del lenguaje, logrando un tratamiento deficiente e integral.

Se coordinó con instituciones involucradas en la detección temprana del retraso en el lenguaje, brindando a los padres de familia una orientación adecuada.

\section{Recomendaciones.}

Que la actualización del equipo de salud contribuyan positivamente en el mejoramiento de la salud de los mismos.

Las actividades desarrolladas en el programa lograron integrar de manera participativa a las madres y padres de familia que forman parte de la población. 
Que la actualización del equipo de salud contribuya positivamente en el mejoramiento de la salud de los niños/as y que la información a los padres de familia junto con los servicios que se brindan, para que el niño y familia reciban el apoyo y tratamiento adecuado.

Que las autoridades coordinen con los responsables del área del lenguaje en programas y proyectos en bienestar de los niños/as con retraso en el lenguaje.

\section{Bibliografía.}

Álvarez Gómez MJ, Soria Aznar J, Galbe Sánchez-Ventura J. Importancia de la vigilancia del desarrollo psicomotor por el pediatra de Atención Primaria: revisión del tema y experiencia de seguimiento en una consulta en Navarra. Rev Pediatr Aten Primaria. 2009, Disponible en: http://scielo.isciii.es/scielo.php?script=sci_arttext\&pid=S1139-76322009000100005\&lng=es

Buele M. (2008); Situación de Salud y sus Tendencias- Módulo III.

Buele M. (2010) Guía Didáctica. Reestructurada Diseño, Ejecución y Gerencia de Proyectos para Salud: Trabajo de Grado II- Módulo IV. UTPL

Calderón Astorga N: "Detección de trastornos del Lenguaje". Disponible en:http://www.sld.cu/galerias/pdf/sitios/rehabilitaciontemprana/deteccion

Castejón \& Navas, (2011) Dificultades y trastornos del aprendizaje y del desarrollo en infantil y primaria: Editorial Club Universitario, 2011

Davila, S. (2009); Siete Encadenamientos Estratégicos para hacer Ingeniería de Procesos, Ecuador.

García Martínez C M, López Betancourt M, Dávila Corrales U. Programa de intervención del lenguaje dedicado a educadoras y padres. en: Intervención temprana en niños con retraso del lenguaje. Disponible en: http://www.sld.cu/galerias/pdf/sitios/rehabilitacionlogo/intervencion_temprana_en_ninos_con_retraso_del_lenguaje.pdf

Jiménez S. A. (2009), ,Expresión y comunicación Ciclos Formativos. Servicios socioculturales y a la comunidad

Lantigua Cruz A, Portuondo Sao M, Collazo Mesa T, Lardoeyt Ferrer R. Epidemiology of Prenatal Genetic and Environmental Factors of Mental Retardation in Cuba. MEDICC Review International Journal of Cuban Health and Medicine. 2008; 10 (1). Disponible: en: http://www.sld.medicc.org/mediccreview/index.php?issue=5\&id=48\&a=vahtml

Licha, I. (2009). Herramientas para la formación de políticas. El análisis de los actores. Fondo España PNUD. 
López Betancourt M.: "Retraso del lenguaje". Disponible en:http://www.sld.cu/galerias/pdf/sitios/rehabilitaciontemprana/retraso_del_lenguaje_1

López P, Ortiz C \& Mldes Viviana (2008) Terapia Ocupacional en la Infancia: Teoría y Práctica. Colección Panamericana de terapia ocupacional: Panamericana, 2008

Machin Verdes M, Puron Sopena E, Castillo Mayedo JA. Reflexiones sobre la intervención temprana en niños con síndrome de down considerando la familia y la comunidad. Revista haban cienc méd. 2009; Disponible en:http://scielo.sld.cu/scielo.php?script=sci_arttext\&pid=S1729519X2009000100002\&lng=es\&nrm=iso . accedido en 6 de marzo 2011.

Marín, A., Jaramillo, B., Gómez, R., Góme,z U.(2008 ) Manual de Pediatría Ambulatoria. Colombia: Ed. Médica Panamericana

Martínez R. (2010). La Coherencia y la Coordinación de las Políticas Públicas. Aspectos Conceptuales y Experiencia. En Jefatura de Gabinete de Ministros de la Presidencia de la Nación, Los desafíos de la coordina ción y la integralidad de las políticas y gestión pública en América Latina, Proyecto de Modernización del Estado. Ciudad Autónoma de Buenos Aires.

Peden M. (2008). World report on child injury prevention. Ginebra, Organización Mundial de la Salud, y Nueva York, Fondo de las Naciones Unidas para la Infancia.

Piedra, M. (2010) Reestructurada: Guía Didáctica Dra. Buele Maldonado, Norita; Diseño, Ejecución y Gerencia de Proyectos para Salud- Módulo IV.UTPL

Ramos M. (2009); Gerencia Integral en Salud- Módulo I.

Rossel C. Katherine. «Apego y vinculación en el síndorme de Down. Una emergencia afectiva». Revista Pediatría Electrónica. 2004; en: http://www.revistapediatria.cl/vol1num1/3.htm. Consultada 6 de diciembre de 2011.

Salas G. \& Lizama E. (2009). Historia de la Psicología en Chile. Editorial Universidad de La Serena.

Trias. D. \& Cuadro, A (2008) (Remitido). Desarrollo de la conciencia fonémica: evaluación y seguimiento de un programa de intervención. Rev. Sociedad de Neuropsicología Argentina.

Unicef (2012) Coordinación de políticas, programas y proyectos sociales. Disponible en: http://www.unicef.org/argentina/spanish/cippec_uni_coordina_politicas.pdf 